\title{
Efficient Methods to Select Top-K propagators based on Distance and Radius Neighbor
}

\author{
Mohammed Alshahrani \\ Computer School, Wuhan University, \\ Wuhan, Hubei, China
}

\author{
Fuxi Zhu \\ Computer School, Wuhan University \\ Wuhan, Hubei, China \\ fxzhu@whu.edu.cn
}

\author{
Mervat Bamiah \\ College of Computer, Prince Sultan \\ University, Riyadh, Saudi Arabia
}

\author{
Sheng Huang \\ Computer School, Wuhan University \\ Hubei, China \\ Soufiana Mekouar \\ University, Rabat, Morocco
}

\section{Keywords}

Radius-Neighborhood Degree; Top-K propagators; Heuristics algorithms; Influence maximization.

\section{INTRODUCTION}

The social network is an abstract presentation of social systems where users diffuse, share and adopt ideas and information through these social platforms such as Facebook, Twitter, and LinkedIn which have experienced explosive use and popularity. These social online platforms provide users with an unprecedented opportunity to communicate and share ideas and information. Additionally, social networks have attracted companies to exploit the social system's data to analyze the market and promote products to potential customers. As these platforms play a fundamental role in spreading the information by extending the relationship network of each entity, this may increase the effect of influence spread in a connected network. Moreover, designing a good selection of Top-K propagators (i.e. influential users) approach is of great importance for influence to reach a numerous number of users within the social platforms. This spread of influence can be observed in real life when a rumors or ideas gain a lot of attention through the effect of word of mouth, advertising, and social media. These phenomena have attracted the attention of the scientific community from various fields including social science [1-2], computer science [3-5] and biological networks [6-8].

Several related works have been performed to analyze and improve the cascading process within the network. Various approaches have been proposed to solve the problem of identifying Top- $\mathrm{K}$ most influential users (referred to as Top- $\mathrm{K}$ propagators). Kemp et al. [3] gave relatively good influence spread in a few milliseconds. Their work is the most cited work in the field of influence maximization. They have proposed a greedy algorithm that gives good performance and influence coverage but suffers from high time complexity. Chen et al. [9] stated that finding Top-K influential users on a large graph of 15,000 nodes may take days on a modern server. Not to mention the time taken to identify the Top-K propagators and use the degree centrality. Other research works have been proposed to optimize the runtime of the greedy algorithm such as CELF algorithm [10], CELF++ algorithm [11].

This paper proposes two new algorithms considering the results of [9]. These proposed algorithms are for selecting Top-K propagators based on degree centrality heuristic and distance that separate the selection of each seed node from another within the network. Regarding network topology, diverse network properties 
can be extracted. Nodes and edges information permits to calculate various network metrics that assist to design any model of seed set selection such us size, diameter, degree centrality, identify vertex neighborhood, compute edge weight according to certain criterions, Page Rank centrality and other characteristics of the network. These topological characteristics provide insights of information distributions across the network and extent flows directions, as well as the speed of information that reaches a given range of users. This study considers the good performance of degree centrality measure in terms of influence achieved and low runtime complexity. Furthermore, the in-depth investigation has been performed on the usefulness of this metric. The results of the observation are that the propagation diffusion probability of nodes besides degree centrality plays a critical role in the diffusion process within the network. This paper proposes an effective method to distinguish and identify Top-K propagators in terms of influence coverage, by considering two proposed scoring metrics based on degree centrality to rank nodes from the highest score values to the lowest. The main contributions of this study are as follows:

- Proposing "Radius-Neighborhood Degree" a new metric based on counting the degree centrality of each user from immediate neighborhood to the radius of the network.

- Designing a new algorithm "RND d-hops" based on the proposed metric "Radius-Neighborhood Degree" and separating each selected seed in the queue from another with a certain number of hops. This prevents the selection of seeds in the same neighborhood and reduces the number of selected seeds that may influence the same users while keeping a good influence coverage.

- Proposing "Radius-Weighted Edges" a new algorithm that is based on degree centrality by exploiting edges weight which should be greater than a certain propagation probability threshold.

- Providing a new algorithm "CPRND d-hops" based on two proposed metrics by combining the nodes with highest edges weight obtained by "Radius-Weighted Edges" algorithm and highest "Radius-Neighborhood Degree" metric.

- Conducting extensive experiments on large-scale datasets that demonstrated its performance over state of art algorithms especially in terms of influence achieved.

The rest of this paper is organized as follows. Section 2 presents some related research work performed on the selection of Top-K influential users. Section 3 discusses problem formulation of Top$\mathrm{K}$ influence maximization problem. Section 4 introduces the proposed "Radius-Neighborhood Degree" metric, and an algorithm for selection of Top-K propagators "RND d-hops". Section 5 describes the proposed new algorithm for selection of Top-K propagators "CPRND d-hops" which utilizes the new two metrics. Section 6 views the experiment results which were conducted on large-scale data. Finally, Section 7 concludes the paper with the main finding and set up future research directions.

\section{RELATED WORK}

Various approaches and algorithms have been proposed to solve the problem of influence maximization. This section discusses related works performed on the Top-K influential users' selection to maximize the influence coverage and reduce runtime complexity for improving the scalability and efficiency of influence maximization models. Several researchers tried to improve the efficiency of the greedy algorithm [9-11]. Kempe et al. [3] demonstrated that the influence maximization under the Independent Cascade (IC) model and linear threshold model are NP-Hard. Their greedy algorithm for both models achieves an approximation ratio of 1-1/e. It estimates the influence spread by using Monti Carlo simulation which makes it inefficient. The greedy algorithm proved to achieve the highest influence coverage but suffers from high runtime complexities.

Tang et al. [12] proposed TIM+ algorithm with near-optimal time complexity. They addressed its scalability and efficiency for time complexity by showing its ability to process large-scale graph in one-hour duration. They presented both theory and practice of influence maximization problem. They argued that TIM+ algorithm can handle large graph at a near-optimal time under the IC model but fail to achieve a higher influence spread. TIM+ algorithm proved to have good runtime but gives poor influence spread.

Kimura et al. [13] proposed a bond percolation method based on [3] that can compute efficiently all the marginal gains $\nabla \sigma(\mathrm{A})$ for the influence degree $\sigma(\mathrm{A})$ of a given target set $\mathrm{A}$ and solving the problem under the hill climbing greedy algorithm. They removed edges that do not contribute to information diffusion and perform the propagation on the subnetwork. The aim was to improve the performance of the greedy algorithm, but scalability remained a significant challenge.

Zhou et al. [14] proposed two-stage greedy algorithm by including users' preferences (GAUP) to find Top-K influential users. The algorithm computes nodes preferences on each topic using the SVD-based Latent Semantic Indexing (LSI). Moreover, the IC is accommodated to users' preferences from the first stage, then the greedy algorithm mines top $\mathrm{K}$ influential users on a specific topic.

Qiao et al. [15] proposed a new centrality measure based on the decompositions of the graph into subgraphs and the computation and analysis of the entropy of nodes neighbors. They calculated the influence spread through a path between nodes and their twohop neighbors resulted with equal weight. The local influence is computed from its immediate neighbors while the indirect influence of node is computed through its two-hop neighbors. They assumed that users cannot be affected by three degrees of separation which reduces the computation complexity. Their assumption is interesting as we go further the influence decreases and time complexity reduces. However, sometimes, when nodes are highly ranked and more influential the spread of influence could achieve more than three degrees of separation, this constraint would decrease the influence coverage. Our proposed algorithms did not limit the extent influence may touch other nodes, but instead controls that by identifying ego network for each selected seed by a certain distance which depends mainly on the size of the network and separates the seeds with a certain distance that permits to identify seeds that may influence distinct users.

Bao et al. [16] proposed a method to identify influential spreaders based on a Heuristic Clustering (HC) algorithm. Their algorithm is created on the similarity index to classify nodes into different clusters, then the center nodes in clusters are chosen as multiple spreaders. Their proposed method selects multiple spreaders that should be scattered and be important. Their process concludes selecting a certain number of nodes as initial centers and aggregating each node into corresponding clusters by a certain similarity measure. Then the center node of each cluster is updated in next step. The clustering process is repeated until the 
system is stable, and the centers are chosen as multiple spreaders The proposed algorithm ensures that the selection of multiple spreaders dispersed within the network and avoids the selection of negligible nodes. Their technique is like our proposed algorithms which select seed set dispersed over the network. However, our algorithms employ two efficient metrics to select high ranked nodes and then adopt an efficient method of separating each consecutive choice of seed set, which makes our proposed algorithms better in term of influence coverage.

Kim et al. [17] introduced the Influential Neighbors Selection (INS) problem to select a node's neighbors to efficiently disseminate its information. They presented four selection strategies to select influential neighbors within the network which are degree, random, volume and weighted-volume strategies. Then, they discussed which selection methods are recommended under which conditions in short and long-term propagation. They found that it is beneficial to use the degree selection strategy for short-term propagation while using random selection strategy for long-term propagation.

Zhang et al. [18] proposed a new method to select Top-K influential users under the SIR model that acts as the IC model in influencing users when fixing the recovery rate to 1 and the probability of activation to a constant in SIR model. They tried to select Top-K propagators based on vote received by node neighbors and the vote that a node gives to its neighbors. Each node holds two vote values the first is the voting ability to spread the influence on other node neighbors. and a score value based on the sum of all neighbors voting abilities. Both Top-K selection methods in [17-18] provide an interesting impact on influence coverage.

Nguyen et al. [19] faced difficulty identifying voting neighbor's abilities since this information is not available on all networks. the traditional selection methods such as degree and random may fail alone to identify Top- $\mathrm{K}$ propagators and may have random strategy selecting low ranked nodes as seeds set. Our algorithms avoid the poor selection by identification of high ranked nodes according to the proposed metrics that permits to obtain a high quality of seed set selection dispersed over the network.

\section{PROBLEM FORMULATION}

The social network has been considered in this study as an undirected graph $\mathrm{G}=(\mathrm{V}, \mathrm{E})$, where $\mathrm{V}$ represents a set of users and E refers to a set of relationships that link users through friendship, kinship, co-authorship or professional relationship in social platforms. All these relationships types may be influenced by users who invite neighbors to adopt the desired behavior with high rate. We represent the number of users in a network as $n=|\mathrm{V}|$ and the number of edges as $\mathrm{m}=|\mathrm{E}|$. In this experiment, it is assumed that nodes which have the highest score value of centrality measures are more likely to influence numerous users. This is due to the tendency of users to follow and adopt actions of known users who have the highest centrality score. From this observation, the choice of initial propagators is a vital step to design an effective diffusion model, whereby, when selecting such initial influential users, the influence coverage will be maximized within the network. Kempe et al. [3] demonstrated that a high degree heuristic provides a relatively large influence spread and a low running time complexity compared to other centrality-based heuristics which give a lower influence spread and require a high time complexity. Chen et al. [9] argued that using a heuristic for an IC model with small propagation probability match the influence spread of the greedy algorithm proposed in [3] which provides the highest influence coverage while suffering from a high running time complexity.

This study considers the results obtained by $[3,9]$ in terms of matching the influence spread of the greedy algorithm and the low running time of degree discount heuristic compared with high computation complexity of the greedy algorithm, have proposed two new algorithms to select Top-K propagators under the IC model using two new metrics based mainly on degree centrality. The study starts by introducing the problem formulation of the Influence maximization and then proposing "RND d-hops" new algorithm which selects the node that has the highest score of the newly proposed metric "Radius-Neighborhood Degree".

Definition 1: We define a sample size $\mathrm{K}$ as Top-K influential users (Top-K propagators), the users who have the high score of centrality metrics in which when targeting such sample or Top-K propagators, the influence coverage will be maximized.

The identification of Top-K influential nodes (propagators) in networks has attracted the attention of the scientific community since its formulation by Domingos and Richardson [20]. The problem of influence maximization is finding the Top- $\mathrm{K}$ propagators and applying a cascading model that can be formalized as follows:

- Considering a network that includes $\mathrm{n}$ users linked by $\mathrm{m}$ relationships.

- Designing seeds set selection method as initial seeds set $\mathrm{S}$ of initially active nodes.

- $\quad$ Generate a cascade of average size $R=\operatorname{RndIC}(S)$.

- Starting the spreading process.

In this study, IC model is used to measure the influence spread, which was adopted by $[3,9]$, where we obtain a seed set generated from our two new proposed metrics. Then those seeds set are used as an input in the IC model to measure the influence spread of a given network. In the following, we recall how users get infected under the IC model. The RndIC defined above presents the used IC model. After identification of seeds set S, the IC is performed on all algorithms presented in this paper with small propagation probability $p$ and the spread follows a uniform random distribution. The same IC model is executed as well on the proposed algorithms that were compared also with [9]. The aim was to identify the best methods for NetHept dataset in which the proposed algorithms outperform the existing approaches.

The IC model used proceeds by assuming that a node $v$ will be influenced by its neighbors with a diffusion probability $p_{u, v}$ which indicates the probability of infection of $v$ by $u$. The IC runs in discrete time step. Some nodes are identified to be active nodes that have already adopted the information. At each step, each activated node will attempt to influence its inactivated neighbors. Each attempt to activate node is performed once, if the active node fails to activate one of its neighbors, then there will be no further possible attempt to activate. The process continues till there is no further possible activation. We provide the formulae of the IC model as stated in [9]:

$$
p_{u, v}=1-(1-\mathrm{p})^{\mathrm{w}}
$$

Where (p) refers to uniform propagation probability. We take a small propagation probability as [3,9]. And $w$ represents the number of edges between two users. We start by recalling the concept of degree centrality which is a robust centralization metrics from network science used for influence maximization in various research papers[9][21][22]. The high degree centrality 
provides a user with a special potential to influence other users and incite them to imitate his/her behavior. In network science, the degree centrality of a user $u$, is defined by the number of adjacent users that hold a specific relationship with user $u$. The degree centrality denoted as $\operatorname{deg}(u)$ of a user $u$ in a network that contains $n$ users, is given in equation (1) as follows:

$$
\operatorname{deg}(u)=\operatorname{sum}(\operatorname{neig}(u))=\sum_{j}^{n} A(i, j)
$$

The degree centrality empowers the user influence which aims to increase the influence locally, the best way is to exploit the degree centrality metric to rise the adoption of ideas that we spread across the local network. As stated earlier the degree centrality provides good results of influence achieved. However, sometimes a high degree node may not have neighbors with high degree connectivity. This motivated us to propose a new metric "RadiusNeighborhood Degree" centrality $g_{r_{-h o p s}}$. This metric is a straightforward that relies on the computation of each node $u$ degree from immediate neighbors to far away neighbors r-hops distance., the $d e g_{r_{-h o p s}}$ can be computed for each node $\mathrm{u}$, as follows:

$$
\operatorname{deg}_{r_{-h o p s}}(u, r)=\sum_{x \in n e i g\left(u, r_{h}=1\right)}^{r} \operatorname{deg}(x)
$$

Whereby the neig $\left(u, r_{h}\right)$ represents all neighbors of $u$ that are far away $r_{h}$ hops from $u$ with $r_{h}$ hops. The $r$ represents the radius of the network. The proposed metric firstly identifies $\mathrm{x}$ neighbors of $\mathrm{u}$ from immediate neighbors $\left(r_{h}=1\right)$ to neighbors that are radius far away from $u$. Then, using equation (2), $d e g_{r_{-h o p s}}$ computes the degree of $u$ from 1-hops $\left(r_{h}=1\right)$ to $r_{h}=r$.

\section{TOP-K PROPAGATORS SELECTION}

This section discusses the selection of Top-K propagators based on "Radius-Neighborhood Degree" metric. In this experiment, we provide our new Top-K seeds selection algorithm "RND-d hops" which is based on the Radius-Neighborhood Degree given in equation (2). Furthermore, the consecutive two selected seeds are far away from each other by a predefined distance d-hops.

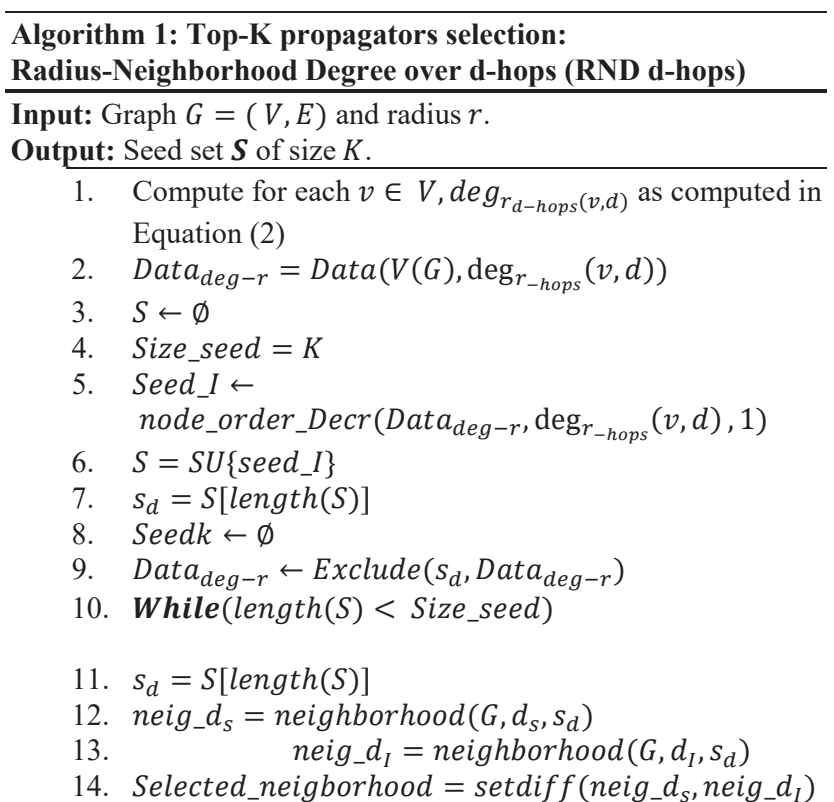

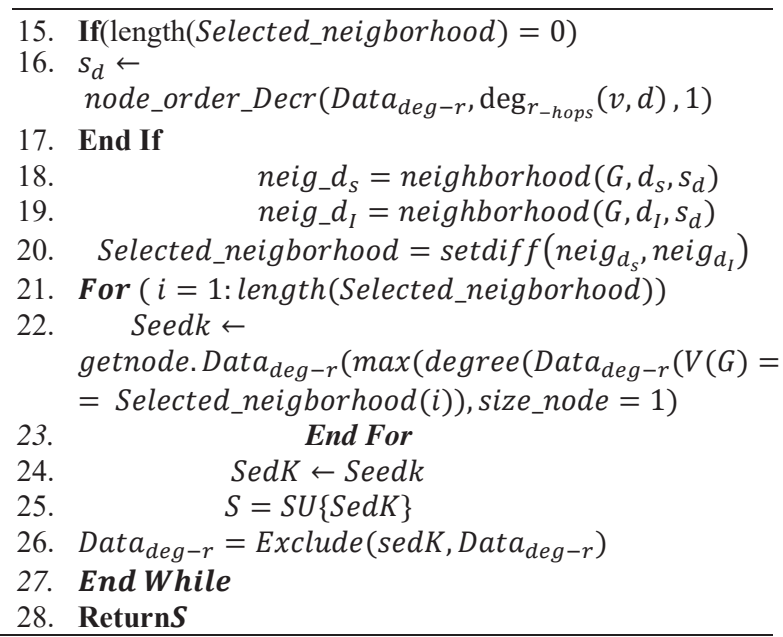

A new probabilistic metric is introduced over node edges. This metric count for each vertex the edges with its neighbors that surpass a pre-computed threshold to the edges of its neighbors which are radius far away from the selected vertex. The main concept of "RND d-hops" first algorithm relies on the computation of each vertex "Radius-Neighborhood Degree" by using equation (2) (Line 1). Then "RND d-hops" algorithm, assigns to each vertex the corresponding Radius-Neighborhood Degree and set the seed set $\mathrm{S}$ to the empty set. The first seed selected by "RND d-hops" algorithm is obtained by ranking data (vertices, Radius-Neighborhood Degree) in decreasing order, then selecting the vertex as a first seed that has the highest RadiusNeighborhood Degree value and fixes the seed set size (Line 2-5). Thereafter, the first selected seed seed_I is added to the seed set $S$ (line 6). Then the algorithm chooses the last seed set $S$ in the queue $s_{d}$ (line 7) and an initialization with an empty set of seed set $S e e d K$ that will include the nodes with the maximum RadiusNeighborhood Degree (Line 8). Then $s_{d}$ is excluded from the data (vertices, Radius-Neighborhood Degree) (Line 9).

The algorithm proceeds by taking the last selected seed in the queue $s_{d}$, a vertex stored in the seed set $S$ (line 11) as an initial seed. Then getting all neighbors or (ego network) of $s_{d}$ that are far away $d_{S}\left(d_{I}\right)$ hops (line 12) and (resp. line 13). The algorithm then excludes redundant vertices and store the non-redundant nodes in Selected_neigborhood (line 14), which will be tested whether it is empty (line 15). In case of empty Selected_neigborhood, the algorithm selects new seed that has the highest radiusneighborhood degree, this is done by assigning the node with the highest score of "Radius-Neighborhood Degree" computed in equation (2) to seed node $s_{d}$ (line 16). The reason for testing Selected_neigborhood is to prevent the selection of a leaf vertex that do not have enough relationships across a radius distance or to prevent the selection of nodes that are in small isolated subgraphs.

Thereafter, the algorithm continues with the same manner as previously (lines 12-14) to compute Selected_neigborhood that will be used to select seed from these selected neighbors that have the highest Radius-Neighborhood Degree (line 21-23). Then, assign the new selected seed denoted Seedk to set seed $S$ and exclude the new selected seed from the data (24-26). This procedure from $(10-27)$ is repeated till the size of seed set is equal to $K$. The algorithm return seed set $S$ of size $K$, and is executed once for all seed set $\mathrm{K}$. For instance, for $K=10,20, \ldots, K_{f}$, the 
algorithm will be executed as explained above from (lines 1-28). For example, for the size seed selection of $K=30$ the algorithm will be executed, from (lines 4-28).

After obtaining the seed set $S$ by "RND d-hops" algorithm, we run the standard IC model with a small propagation probability $p$, to achieve a good influence spread across the network. Since despite designing a metric based on multi-hops degree metric that permits to identify users that are most reachable. There is a need to have knowledge of possibility of infection over edges between pairs of nodes. This probability will be inferred from degree centrality, which makes our approach according to experiments outperforms the state of the art algorithms. As for designing a good selection seed algorithm we did not rely only on centrality measure or structural properties but depended on the ability of the predefined infection probability between individuals that may lead users to adopt the behavior. It is vital to include the infection probability as a characteristic of seed selection besides structural proprieties which improves the efficiency of the proposed seed selection algorithm which is the aim of the algorithm 3 "CPRND-d hops".

\section{SEED SELECTION}

This section presents the seed selection of Top-K propagators based on combined Radius-Neighborhood Degree and RadiusWeighted Edges. The procedure starts with transforming unweighted graph to weighted one, then computing the maximal propagation probability threshold, followed by the introduction of Radius-Weighted Edges metric. The process begins from node $v \in V$, whereby each node $v$ has a relationship with another node $u$ via an edge $(u, v) \in E$. Then computing the degree centrality for each user $v$ using equation(1), as well as computing two weights for each edge. The proposed calculation is based on the degree of each node of an edge over the sum of the degree of nodes that hold each edge. The weight values for each edge weight $_{v, u}^{1}(v)$ and weight $v_{v, u}^{2}(u)$ are computed as follows:

$$
\begin{aligned}
\text { weight }_{v, u}^{1}(v) & =\frac{\operatorname{deg}(v)}{\operatorname{deg}(u)+\operatorname{deg}(v)} \\
\text { weight }_{v, u}^{2}(u) & =\frac{\operatorname{deg}(u)}{\operatorname{deg}(u)+\operatorname{deg}(v)}
\end{aligned}
$$

Next step is obtaining maximal propagation threshold probability denoted as weight $_{\max }(v, u)$ for each edge by taking the max values for each edge as follows:

$$
\text { weight }_{\max }(v, u)=\max \left(\text { weight }_{v, u}^{1}(v), \text { weight }_{v, u}^{2}(u)\right)
$$

From the above equation (3), the maximal propagation probability value can be obtained for each edge, which is required to initiate the diffusion process. For instance, the maximal probability threshold that permits the edges selection with the highest probability of infection and more tendency to adopt the behavior. Each edge in graph $G=(V, E)$ is assigned a maximal weight computed below in equation (4) and obtaining weighted maximal graph denoted as $G^{\prime}=\left(V, E\right.$, weight $\left.t_{\max }\right)$. Next is determining the threshold propagation probability in which the infection may occur or not. This could be done by testing if each edge value in weighted graph $G^{\prime}$ is above the maximal threshold propagation probability value.

\subsection{Maximal propagation probability threshold}

The structural properties such as degree identify the user who has the highest number of neighbors in one hop. However, it is unable to determine the link that spread the influence. Adding a certain propagation threshold of accepting an information would increase by approximately $20 \%$ the spread of influence. However, an active user can influence inactive neighbor if its probability of influence surpasses or is equal to a threshold value that determines if a user will adopt the information or not. The propagation probability threshold is computed by taking the mean of maximum infection probability which can be written as follow:

$$
t h_{\text {infec }}=\frac{\sum_{e=1}^{|E|} P(e)}{|E|}, e \in E
$$

The maximum infection probability threshold can be obtained as follows:

$$
t h_{\text {infec }}^{\max }=\frac{\sum_{e=1}^{|E|} \text { weight }_{\max }(e)}{|E|}
$$

Where $e$ represents an edge in the network.

$|E|$ : represents the total number of edges in the network.

weight $\max _{\max }(e):$ represent the maximum probability of infection between two adjacent users. The number of links that has a probability of infection superior or equal to the propagation probability threshold is counted for each user. This number can be computed by identifying neighbors of the user $u$ and then testing each link between the user $u$ and its neighbors $x i$, if it surpasses the threshold probability.

$$
\begin{gathered}
N b_{\text {prb }}(u)=\operatorname{count}\left(\text { weight }_{\max }\left(u, x_{i}\right)>=\text { th }_{\text {infec }}^{\max }\right), x_{i} \\
=n_{1}, \ldots, n_{x_{n}}
\end{gathered}
$$

Where $x_{i}$ is neighbor of user $u$ and $x_{n}$ is the number of neighbors of user $u$.

\subsection{Radius-Weighted Edges Metric}

This subsection introduces the new proposed metric "RadiusWeighted Edges" that computes the $N b_{p r b}(u)$ for each user $u$, over network radius. The number of links that exceeds the maximal propagation probability threshold in one-hop is computed in equation (5), and the links which have weight superior to maximal threshold propagation probability. Algorithm 2 "Radius-Weighted Edges" computes the number of links of vertex $v$ that surpass the maximal threshold propagation probability across radius hops (number of links starting from vertex $v$ to radius of the network), that satisfies the condition stated in algorithm 2(lines 5-6).

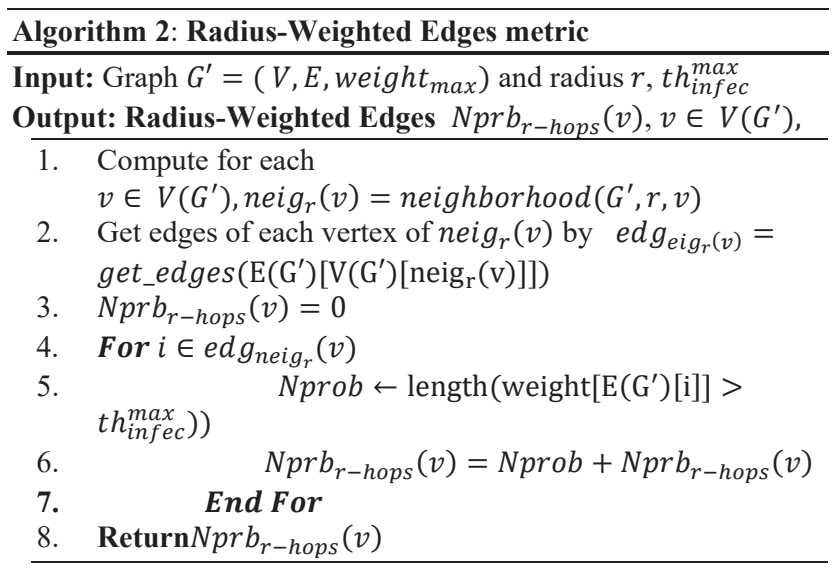




\subsection{Seed selection Top-K propagators algorithm "CPRND d-hops"}

Next step is to design the proposed algorithm 3 for Top-K propagators selection based on combined "Radius-Weighted Edges" and Radius-Neighborhood Degree over $d_{S-I}-h o p$. This facilitates the use of them to select most influential users, which adds strength to the selection process of Top-K propagators. Algorithm 3 "CPRND d-hops", selects the Top-K influential users based on users' Radius-Weighted Edges over $d_{S-I}$ hops away from each seed set S. The procedure is similar to "RND d-hops" algorithm. The difference is that algorithm 3 starts with initial seed selection by computing "Radius-Weighted Edges" for each node applying algorithm 2 , and then creating data by assigning each vertex $v$ the corresponding Radius-Weighted Edges and rank in decreasing order according to the metric proposed in algorithm 2 (lines 1-2). The remaining explanation of algorithm 3 is the same as presented in algorithm 1. The difference is that in algorithm 3, we mix between using radius- neighborhood degree that permits to select the most reachable nodes and RadiusWeighted Edges which assures more efficient way to select users who may adopt the information. Equivalently the computation of seed set $\mathrm{S}$ is the same as explained in algorithm 1 .

\section{Algorithm 3: Combined Radius-Neighborhood Degree and radius-propagation probability threshold neighborhood-based d-hops (CPRND d-hops)}

Input: Graph $G^{\prime}=\left(V, E\right.$, weight $\left.t_{\text {max }}\right)$ and radius $r$.

Output: Seed set $\boldsymbol{S}$ of size $K$.

1. Compute for each $v \in V, \operatorname{deg}_{r-h o p s(v, r)}$ as computed in Equation (2) and $N p r b_{r}-h o p s(v, r)$ as computed in algorithm 2 "radius-weighted edges"

2. Data $_{\text {deg-r, } n p r b-r}=$ $\operatorname{Data}\left(V\left(G^{\prime}\right), \operatorname{deg}_{r-h o p s}(v, r), N p r b_{r}-\operatorname{hops}(v, r)\right)$

3. $S \leftarrow \varnothing$

4. Size_seed $=K$

5. Seed_I $\leftarrow$ node_order_Decr $\left(\right.$ Data $_{\text {deg-r,nprb-r }}, N_{p r b}-$ $\operatorname{hops}(v, r), 1)$

6. $S=S U\{$ seed_I $\}$

7. $s_{d}=S[$ length $(S)]$

8. Seedk $\leftarrow \varnothing$

9. Data $a_{\text {deg-r,nprb-r }} \leftarrow \operatorname{Exclude}\left(s_{d}\right.$, Data $\left.a_{\text {deg-r,nprb-r }}\right)$

10. While $($ length $(S)<$ Size_seed $)$

11. $s_{d}=S[\operatorname{length}(S)]$

12. neig_d $_{s}=$ neighborhood $\left(G^{\prime}, d_{s}, s_{d}\right)$

13. neig_d $d_{I}=$ neighborhood $\left(G^{\prime}, d_{I}, s_{d}\right)$

14. Selected_neigborhood $=\operatorname{setdiff}\left(\right.$ neig $_{d_{S}}$, neig $\left._{d_{I}}\right)$

15. If(length(Selected_neigborhood $)=0$ )

16. $s_{d} \leftarrow$ node_order_Decr $\left(\right.$ Data $_{\text {deg-r,nprb-r }}, N$ Nr $b_{r}-$ $\operatorname{hops}(v, r), 1)$

17. End If

18. $\quad$ neig_d $_{s}=$ neighborhood $\left(G^{\prime}, d_{s}, s_{d}\right)$ 19.

20. Selected_neigborhood $=\operatorname{setdiff}\left(\right.$ neig $_{d_{s}}$, neig $\left.g_{d_{I}}\right)$

21. For $(i=1$ : length(Selected_neigborhood $)$ )

22. $\quad$ Seedk $\leftarrow$

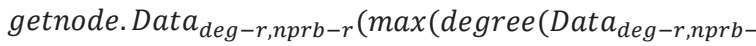
$=$ Selected_neigborhood $(i))$, size_node $=1$ ) End For
26. Data $_{\text {deg-r, } n \text { prb }-r}=$ Exclude (sedK, Data deg-r,nprb-r)
27. End While
28. ReturnS

\section{EXPERIMENTAL RESULTS}

This section presents the proposed conducted experimental results. Large datasets on academic collaboration network NetHept and NetPhy based on [9] were used for testing purposes. The information of the datasets is summarized in Table 1 . The nodes represent the authors, and the edges represent authors of a paper. The graph refers to a multigraph network in which two authors can co-author more than one research paper. The datasets contain many isolated subgraphs (connected components).

Table 1: Description of Datasets

\begin{tabular}{|c|c|c|c|c|}
\hline Datasets & $\mathbf{n}$ & $\mathbf{m}$ & $\mathbf{r}$ & $\begin{array}{c}\text { Number of Connected } \\
\text { Components }\end{array}$ \\
\hline NetHept & 15233 & 58891 & 7 & 1781 \\
\hline NetPhy & 37154 & 231507 & 6 & 3878 \\
\hline
\end{tabular}

\subsection{Existing Algorithms}

This section presents the comparison results of "RND d-hops", "CPRND d-hops" with existing algorithms including degree discount heuristics [9], BCT algorithm [19], TIM+ algorithm [12]. and baselines heuristics that were compared in $[3,9]$. The related algorithms are defined as follows.

- Degree: The degree centrality that selects Top-K propagators with the highest degree centrality computed in equation (1). This baseline heuristic was used for comparison purpose in various research work such as [3] and [9].

- Page Rank: It is used by Google search engine. Page Rank proceeds by counting the number and quality of links of a node to all other nodes in such a way to determine how important is the node. Each node depends on the PageRank of all other nodes [23].

- Degree Discount Heuristic: It was introduced in [9] selects seeds based on the degree centrality score and discount the edge that bond with the next selected seed from the nodes degree computation.

- BCT Algorithm: It was proposed in [19] to find the most cost-effective seed users who can influence the most relevant users to the advertisement.

- TIM+ Algorithm: It was proposed in [12] to improve the scalability of time complexity while providing low coverage spread of influence.

\subsection{Evaluation of Proposed Algorithms Performance}

This section presents the evaluation of "RND d-hops" and "CPRND d-hops" algorithms performance and influence coverage compared with existing algorithms. All algorithms were run under IC model with $p=0.1$. All experiments are performed on a server (ppted' ${ }^{\prime}$ eon ${ }^{\circledR}$ with $2.50 \mathrm{GHZ} 16$ cores, and $120 \mathrm{G}$ memory. The seed size $K$ varies from 10 to 50 for all algorithms. $d_{s}-d_{I}=4$, represents the number of hops between first selected seed and the subsequent seed that will be chosen. The value of $d_{s}-d_{I}$ excludes just immediate neighbors and consider the selection of 
next node seed from all nodes that are within 4-hops away distance.

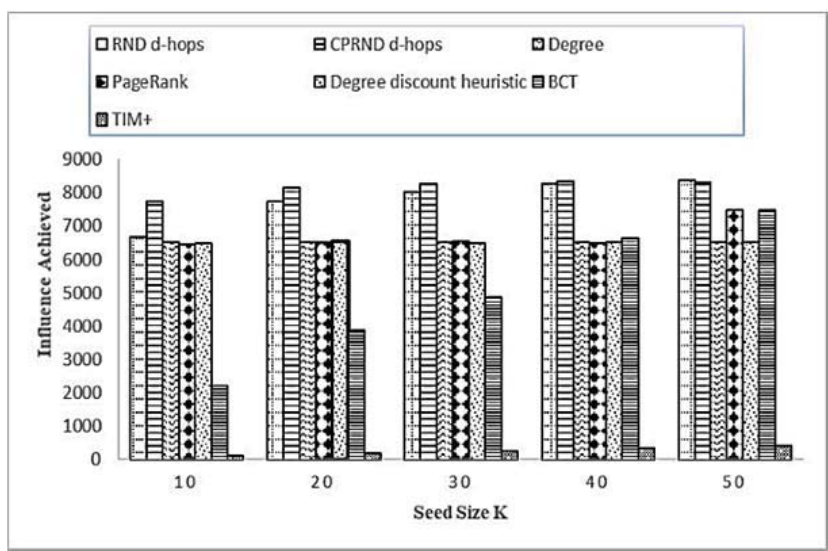

Figure 1. Influence coverage comparisons.

Figure 1, shows the influence coverage achieved by the proposed algorithms compared with the existing algorithms listed in 6.1 and some heuristics on NetHept data under the IC model. It is observed clearly that the proposed algorithms "RND d-hops" and "CPRND d-hops" outperform the existing approaches in terms of influence coverage for all seed size $\mathrm{K}$. This is due to targeting the nodes with highest "radius- neighborhood degree", "RadiusWeighted Edges" metrics and the efficient strategy of selected seed set which is separated by a certain distance. This methodology makes "RND d-hops" and "CPRND d-hops" algorithms efficient in terms of reaching numerous users than the degree discount heuristic, degree heuristic, Page Rank, BCT, $\mathrm{TIM}+$ algorithms.

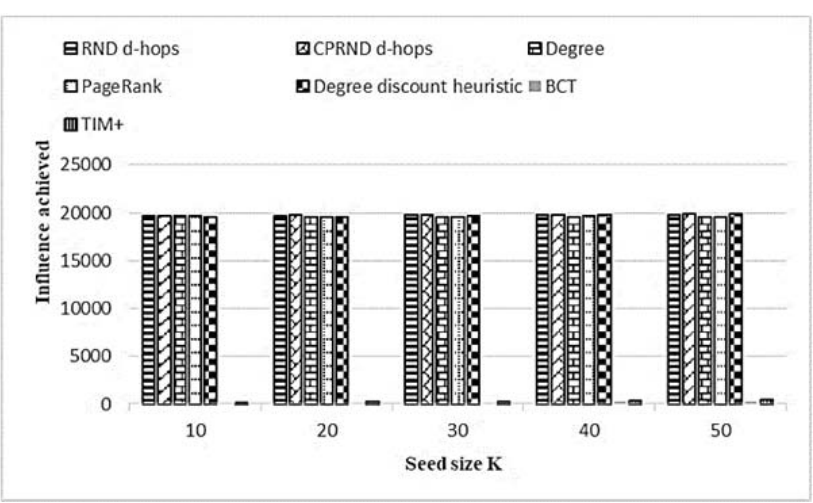

Figure 2. Proposed algorithms influence compression.

Figure 2, shows the influence spread of the proposed algorithms on NetPhy data compared with existing algorithms under the IC model. It is observed that the two proposed algorithms outperform all existing algorithms except for $\mathrm{K}=50$, in which is a little bit lower by $0.78 \%$ for "CPRND d-hops", and lower by $1.41 \%$ for "RND d-hop" than the degree discount heuristic. This could be justified by the fact that setting exact value of distance which separates the seed selection is very important. Indeed, our two proposed algorithms have proved through experiments that they assure a higher influence than the existing approaches. In overall when comparing the influence for the best existing algorithm on all seed set, our "CPNRD d-hops"(Resp. "RND d-hop") algorithm outperforms the discount degree heuristic by $3.74 \%$ (resp. by $1.18 \%)$.

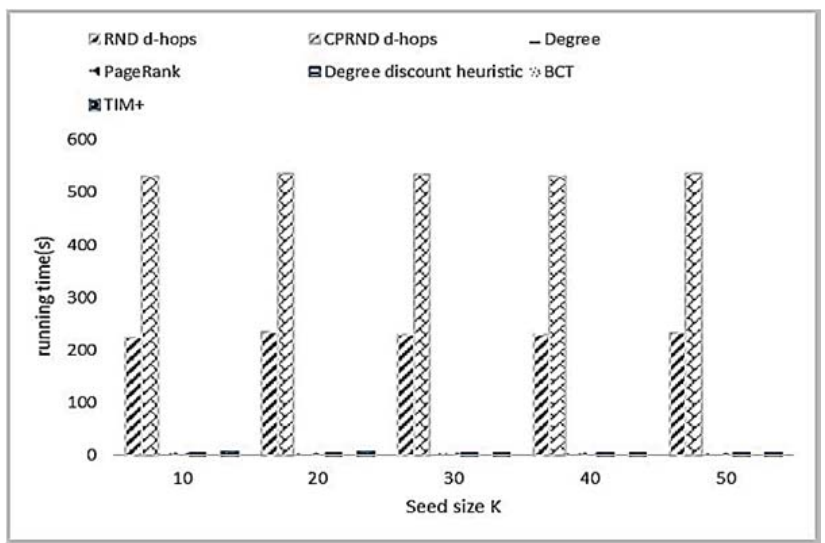

Figure 3. Proposed Algorithm runtime comparison on NetHEPT data under the IC model.

Figure 3 and Figure 4, present the running time of "RND d-hops" and "CPRND d-hops" algorithms on NetHept and NetPhy data respectively compared with existing algorithms presented in this experiment. It is observed that "RND d-hops" and "CPRND dhops" algorithms consume more time than the existing algorithms since they compute some structural properties besides the ego network for each selected seed, as well as the computation of selection metrics over radius distance. Moreover, they attempt to influence more users' that increases slightly the running time. Furthermore, the seed size is selected from a certain distance which is obtained by computing the neighborhood at each attempt of seed selection. Despite "RND d-hops" and "CPRND d-hops" algorithms taking more time than other algorithms, they are still effective in terms of influence coverage which is the aim of influence maximization and could run on the large graph which makes it adequate to a large graph. In summary, "RND d-hops" and "CPRND d-hops" algorithms have proven through experiments a good influence spread compared to the existing approaches. The only exception is for the NetPhy data which gives a little lower influence, this could be justified by the choice of distance that separates each selected seed from another seed, this distance should be controlled by other constraints link constraint threshold that controls the choice of seed set.

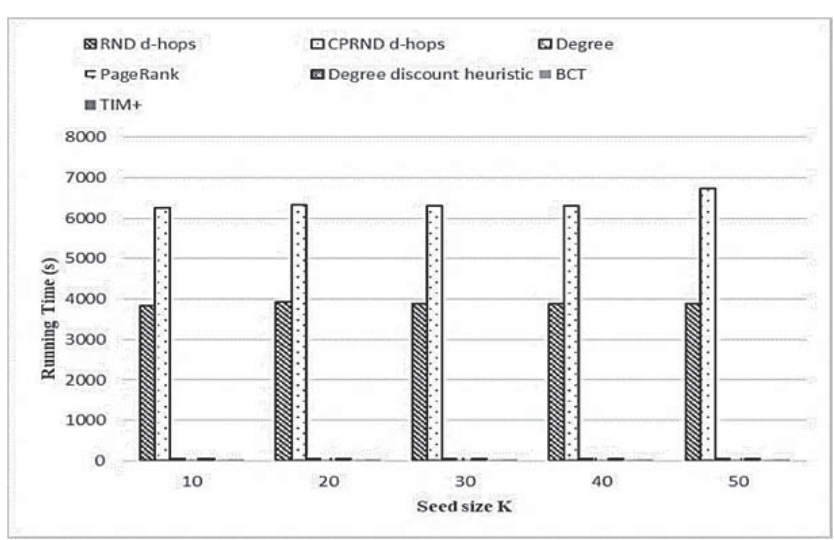

Figure 4. Proposed Algorithm runtime comparison on NetPhy data under the IC model

\section{CONCLUSION AND FUTURE WORK}

This paper presented two new algorithms for Top-K propagators selection. "RND d-hops" algorithm extracts Top-K influential users based on "Radius-Neighborhood Degree" metric. This technique outperforms other techniques such as discount degree, 
Page Rank, degree and other baseline algorithms that consider only node characteristics without separating the seeds set by a certain distance. The second algorithm "CPRND d-hops" identifies Top-K propagators based on the distance. Moreover, the study also proposes "Radius-Weighted Edges" metric and "Radius-Neighborhood Degree" metric. Also, this study proposed new "Radius-Neighborhood Degree" metric which is based on degree centrality, as well as a new "Radius-Weighted Edges" metric, which identifies the edges that have a high possibility of spreading information. The new metrics besides an efficient separation of selected seeds set by a given number of hops were used in the proposed algorithms for the selection of Top-K propagators. Furthermore, the study identified that the proposed algorithms "RND d-hops" and "CPRND d-hops", achieved higher influence coverage compared with existing algorithms in terms of influence spread except only for Netphy data that achieved lower influence than the degree discount heuristic. This is due to the choice of exact distance that separates selected seeds set, which should be addressed in-depth in a future extension of this work. "RND d-hops" and "CPRND d-hops" can be extended easily to other diffusion models as well as an improvement of time complexity is planned to be optimized in the future.

\section{ACKNOWLEDGMENTS}

This research is supported by The National Natural Science Foundation of China with Grants No: 61272277 and 91746206. We thank Alibaba Cloud Private Co., for their technical support.

\section{REFERENCES}

[1] Moscovici, S., Sherrard, C., and Heinz, G. 1976. Social influence and social change. 9(4), 441-446. London: Academic Press. DOI: 10.1002/ejsp.2420090410

[2] Borgatti, S. P., Mehra, A., Brass, D. J., and Labianca, G. 2009. Network analysis in the social sciences. science, 323(5916), 892-895. DOI: 10.1126/science.1165821.

[3] Kempe, D., Kleinberg, J., and Tardos, É. 2003. Maximizing the spread of influence through a social network. In Proceedings of the ninth ACM SIGKDD international conference on Knowledge discovery and data mining. ACM. 137-146.

[4] Chen, W., Wang, C., and Wang, Y. 2010. Scalable influence maximization for prevalent viral marketing in large-scale social networks. In Proceedings of the 16th ACM SIGKDD international conference on Knowledge discovery and data mining. ACM. 1029-1038.

[5] Zhuang, H., Sun, Y., Tang, J., Zhang, J., and Sun, X. 2013. Influence maximization in dynamic social networks. In Data Mining (ICDM), 2013 IEEE 13th International Conference on. IEEE. 1313-1318.

[6] Vitkup, D., Kharchenko, P., and Wagner, A. 2006. Influence of metabolic network structure and function on enzyme evolution. Genome biology, 7(5), R39. DOI: $10.1186 / \mathrm{gb}-$ 2006-7-5-r39.

[7] Friedman, N. 2004. Inferring cellular networks using probabilistic graphical models. Science, 303(5659), 799-805. DOI:10.1126/science.1094068.

[8] Pál, C., Papp, B., and Lercher, M. J. 2005. Adaptive evolution of bacterial metabolic networks by horizontal gene transfer. Nature Genetics, 37(12). DOI:10.1038/ng1686.

[9] Chen, W., Wang, Y., and Yang, S. 2009. Efficient influence maximization in social networks. In Proceedings of the 15th
ACM SIGKDD international conference on Knowledge discovery and data mining. ACM. 199-208.

[10] Leskovec, J., Krause, A., Guestrin, C., Faloutsos, C., VanBriesen, J., and Glance, N. 2007. Cost-effective outbreak detection in networks. In Proceedings of the 13th ACM SIGKDD international conference on Knowledge discovery and data mining. ACM. 420-429.

[11] Goyal, A., Lu, W., and Lakshmanan, L. V. 2011. Celf++: optimizing the greedy algorithm for influence maximization in social networks. In Proceedings of the 20th international conference companion on World wide web. ACM. 47-48.

[12] Tang, Y., Xiao, X., and Shi, Y. 2014. Influence maximization: Near-optimal time complexity meets practical efficiency. In Proceedings of the 2014 ACM SIGMOD international conference on Management of data. ACM. 75 86.

[13] Kimura, Masahiro, Kazumi Saito, and Ryohei Nakano. 2007. Extracting influential nodes for information diffusion on a social network. AAAI'07 Proceedings of the 22nd national conference on Artificial intelligence. ACM. 2, 1371-1376. ISBN: 978-1-57735-323-2.

[14] Zhou, J., Zhang, Y., and Cheng, J. 2014. Preference-based mining of top-K influential nodes in social networks. Future Generation Computer Systems, 31, 40-47. DOI: 10.1109/TrustCom.2011.209.

[15] Qiao, T., Shan, W., and Zhou, C. 2017. How to Identify the Most Powerful Node in Complex Networks? A Novel Entropy Centrality Approach. Entropy, 19(11), 614.

[16] Bao, Z. K., Liu, J. G., and Zhang, H. F. 2017. Identifying multiple influential spreaders by a heuristic clustering algorithm. Physics Letters A, 381(11), 976-983.

[17] Kim, H., and Yoneki, E. 2012. Influential neighbors' selection for information diffusion in online social networks. In Computer Communications and Networks (ICCCN), 2012 21st International Conference on. IEEE. 1-7.

[18] Zhang, J. X., Duan-Bing Chen, Q. D., and Zhao, Z. D. 2016. Identifying a set of influential spreaders in complex networks. Scientific reports, 6 .

[19] Nguyen, H. T., Dinh, T. N., and Thai, M. T. 2016. Costaware targeted viral marketing in billion-scale networks. In Computer Communications, IEEE INFOCOM 2016-The 35th Annual IEEE International Conference on. IEEE. 1-9.

[20] Richardson, M., and Domingos, P. 2002. Mining knowledge-sharing sites for viral marketing. In Proceedings of the eighth ACM SIGKDD international conference on Knowledge discovery and data mining. ACM. 61-70.

[21] Lei, W., Yang, Q., \& Wang, H. 2016. Positive influence maximization algorithm based on three degrees of influence. In International Conference on Intelligent Data Engineering and Automated Learning. Springer, Cham. pp. 503-514.

[22] Kundu, S., Murthy, C. A., \& Pal, S. K. 2011. A new centrality measure for influence maximization in social networks. In International Conference on Pattern Recognition and Machine Intelligence. Springer, Berlin, Heidelberg. pp. 242-247.

[23] Wills, R. S. 2006. Google's page rank. The Mathematical Intelligencer, 28(4), 6-11. 